\title{
MicroRNA-934 promotes colorectal cancer cell proliferation by directly targeting Dickkopf-related protein 2
}

\author{
WEI LIU, LONGAN MA and JIE ZHANG \\ Department of General Surgery, Shaanxi Provincial Cancer Hospital, Xi'an, Shaanxi 710061, P.R. China
}

Received July 1, 2020; Accepted July 2, 2021

DOI: $10.3892 /$ etm.2021.10473

\begin{abstract}
Increasing evidence demonstrates that dysregulation of microRNAs (miRNAs/miRs) is implicated in the development of colorectal cancer. However, the biological functions of several differentially expressed miRNAs remain unknown. In the present study, a bioinformatic analysis of a previously published microarray data and reverse transcription-quantitative PCR analysis demonstrated that miR-934 expression was upregulated in colorectal cancer samples collected from patients. Mechanistically, Dickkopf-related protein 2 (DDK2) was identified as a novel target gene of miR-934 in colorectal cancer cells. Knockdown of DDK2 reversed the inactivation of Wnt signaling pathway induced using miR-934 inhibitor in colorectal cancer cells. In addition, DDK2 silencing reversed miR-934 inhibitor-induced cell proliferation inhibition and elevation of cell apoptosis. The results demonstrated that DDK2 mRNA expression was negatively associated with miR-934 expression in colorectal tumors. Collectively, the results of the present study demonstrated that the miR-934/DDK2 axis regulated colorectal cancer cell proliferation, suggesting that miR-934 may be a biomarker for patients with colorectal cancer.
\end{abstract}

\section{Introduction}

Globally, colorectal cancer was reported as the third most commonly diagnosed cancer type for men and the second most commonly diagnosed cancer type for women in 2018 (1). An estimated 551,000 patients died from colorectal cancer in 2018 , which accounts for $>5 \%$ of cancer-associated mortality worldwide (1). Despite advancements in treatment strategies, the prognosis of patients with colorectal cancer remains poor, with a 5-year overall survival rate of $\sim 40 \%$ (2). Several novel therapeutic approaches, such as aspirin-based chemoprevention

Correspondence to: Dr Jie Zhang, Department of General Surgery, Shaanxi Provincial Cancer Hospital, 309 Yanta West Road, Xi'an, Shaanxi 710061, P.R. China

E-mail: zhangjiespc@aliyun.com

Key words: microRNA-934, colorectal cancer, Dickkopf-related protein 2 and targeting cyclin-dependent kinase 4/tyrosine-protein kinase Fyn have improved the treatment efficacy (3-5). However, the molecular mechanism contributing to colorectal cancer progression remains elusive, making it difficult to develop therapeutic approaches for patients. Thus, it remains critical to determine the molecular mechanism to provide novel targets for the treatment of colorectal cancer.

MicroRNAs (miRNAs/miRs) are non-coding, single-stranded, short molecules ubiquitously expressed in human cells (6). miRNAs degrade mRNAs and inhibit the translation process by directly binding to the complementary sites in the 3'-untranslated region (UTR) of target mRNAs (7). miRNAs regulate several physiological processes and dysregulation of miRNAs results in the development of different types of human diseases (8-11). Aberrant expression or mutation of miRNAs are implicated in the initiation and development of different types of cancer, including colorectal cancer $(12,13)$. For example, miR-145 is downregulated in colorectal cancer, and can directly target transcriptional regulator ERG to suppress migration and invasion of cancer cells (12). Several miRNAs, including miR-934 have been identified as differentially expressed in colorectal cancer based on human miRNA microarray (14). miR-934 is involved in cancer cell proliferation, invasion, drug resistance and apoptosis of ovarian cancer and head and neck squamous cell carcinoma (HNSCC) $(15,16)$. However, to the best of our knowledge, the role of miR-934 in colorectal cancer remains known.

The present study aimed to investigate the biological role of miR-934 in colorectal cancer cells.

\section{Materials and methods}

Patients and materials. A total of 40 colorectal tumor tissues and matched adjacent normal tissues ( $\geq 5 \mathrm{~cm}$ from tumors) were collected from patients (24 males and 16 females; including $12 \mathrm{~T} 1,13 \mathrm{~T} 2,10 \mathrm{~T} 3$ and $5 \mathrm{~T} 4$; age range, 49-72 years; median age, 56 years) who underwent surgery at the Shaanxi Provincial Cancer Hospital (Xi'an, China) between June 2015 and July 2018. None of these patients received chemotherapy or radiotherapy before surgery. Tissue samples were stored at $-80^{\circ} \mathrm{C}$ until subsequent experimentation. The present study was approved by the Ethics Committee of Shaanxi Provincial Cancer Hospital (approval no. 2015-23; Xi'an, China) and written informed consent was provided by all patients prior to the study commencement. 
Cell culture and transfection. The immortalized human colon cell line FHC and human colorectal cancer cell lines HCT116 and HT29 were purchased from the American Type Culture Collection. All cell lines were authenticated via STR profiling and maintained in DMEM supplemented with $10 \%$ fetal bovine serum (both purchased from Gibco; Thermo Fisher Scientific, Inc.) at $37^{\circ} \mathrm{C}$ in an incubator with $5 \% \mathrm{CO}_{2}$.

miR-negative control (NC) mimic, miR-NC inhibitor, miR-934 inhibitor and miR-934 mimic were synthesized and purchased from Suzhou GenePharma Co., Ltd. A total of $50 \mathrm{nM}$ miR-NC mimic (5'-CUAUCCACCAGGUUGCUU UGACC-3'), miR-NC inhibitor (5'-UUGUACUACACAAAA GUACUG-3'), miR-934 inhibitor (5'-CCAGUGUCUCCAGUA GUAGACA-3') or miR-934 mimic (5'-UGUCUACUACUG GAGACACUGG-3') was transfected into HCT116 cells using Lipofectamine ${ }^{\circledR} 3000$ (Invitrogen; Thermo Fisher Scientific, Inc.) according to the manufacturer's instructions. Control small interfering (si)RNA (5'-UAAGGCUAUGAAGAGAUA C-3') and Dickkopf-related protein 2 (DDK2) siRNA (5'-CAG CAGGACGAAUCCAAG-3') were purchased from Suzhou GenePharma Co., Ltd. A total of $30 \mathrm{nM}$ control siRNA or DDK2 siRNA was transfected into HCT116 cells using Lipofectamine 3000 according to the manufacturer's instructions. The reagent was mixed with siRNA or miRNA mimic or miRNA inhibitor at room temperature for $10 \mathrm{~min}$. Cells were subjected to reverse transcription-quantitative (RT-q)PCR and western blot analysis $48 \mathrm{~h}$ post-transfection.

$R T$ - $q P C R$. Total RNA was extracted from cells and tissues using TRIzol ${ }^{\circledR}$ reagent (Invitrogen; Thermo Fisher Scientific) and reverse-transcribed into cDNA using the miScript Reverse Transcription kit (cat. no. 218061; Qiagen $\mathrm{GmbH}$ ), with the following temperature protocol: $37^{\circ} \mathrm{C}$ for $1 \mathrm{~h}$ and $95^{\circ} \mathrm{C}$ for 5 min. qPCR was subsequently performed using the SYBR Premix EX Taq kit (cat. no. DRR041 A; Takara Bio, Inc.), with the following thermocycling conditions: $95^{\circ} \mathrm{C}$ for $2 \mathrm{~min}$; 35 cycles of $95^{\circ} \mathrm{C}$ for $10 \mathrm{sec}$ and $55^{\circ} \mathrm{C}$ for $30 \mathrm{sec}$. The miRNA was amplified with the stem loop primer and universal primers. Relative expression levels were quantified using the $2^{-\Delta \Delta \mathrm{Cq}}$ method (17) and normalized to the internal reference genes U6 (miRNA) and GAPDH (mRNA). The primer sequences used for qPCR are listed in Table I.

RNA immunoprecipitation (RIP). RIP was performed using the Magna RIP RNA-Binding Protein Immunoprecipitation kit (cat. no. 17-700; EMD Millipore), according to the manufacturer's protocol. After washing to remove unbound material, RNA was extracted using TRIzol ${ }^{\circledR}$ reagent. Protein argonaute-2 (AGO2; cat. no. 2897; 1:100) and IgG (cat. no. 3900; 1:100) antibodies were purchased from Cell Signaling Technology, Inc. Harvested RNAs were assessed via RT-qPCR analysis using the aforementioned protocol and primers.

Western blotting. $\beta$-catenin (cat. no. $8480 ; 1: 2,000$ ) and phosphorylated (p)- $\beta$-catenin (Ser675; cat. no. 4176; 1:2,000) antibodies were purchased from Cell Signaling Technology, Inc. GAPDH (cat. no. ab8245; 1:5,000) antibody was purchased from Abcam. c-Myc (cat. no. sc40; 1:2,000) and cyclin D1 (cat. no. sc450; 1:2,000) antibodies were purchased from Santa Cruz Biotechnology, Inc. HRP-conjugated secondary
Table I. Sequences of primers used for quantitative PCR.

Primer Sequence (5'-3')

miR-934 stem-loop

GTCGTATCCAGTGCGTGTCGTGG AGTCGGCAATTGCACTGGATACG ACCCAGTGTC

miR-934 forward GGGTGTCTACTACTGGAGA miR-934 reverse CAGTGCGTGTCGTGGAGT U6 forward GCTTCGGCAGCACATATACTAA AAT

U6 reverse

DKK2 forward

DKK2 reverse CGCTTCACGAATTTGCGTGTCAT $\beta$-catenin forward CCCCACCAAGGATCATCGG CCGGGATGTGAGGGGTTAAGA

$\beta$-catenin reverse CACAAGCAGAGTGCTGAAGGTG c-Myc forward c-Myc reverse cyclinD1 forward cyclinD1 reverse GAPDH forward GAPDH reverse GATTCCTGAGAGTCCAAAGACAG TACCCTCTCAACGACAGCAG TCTTGACATTCTCCTCGGTG CTTCCTCTCCAAAATGCCAG AGAGATGGAAGGGGGAAAGA GTCTCCTCTGACTTCAACAGCG ACCACCCTGTTGCTGTAGCCAA

miR, microRNA.

antibodies against mouse (cat. no. ab205719; 1:10,000) and rabbit (cat. no. ab6721; 1:10,000) were purchased from Abcam.

Protein lysates from colorectal cancer cells were prepared using RIPA lysis buffer (Thermo Fisher Scientific, Inc.) according to the manufacturer's protocol. BCA Protein Assay kit (cat. no. 23225; Thermo Fisher Scientific, Inc.) was used to determine the protein concentration. A total of $20 \mu \mathrm{g}$ proteins per lane were separated using SDS-PAGE ( $8 \%$ gel) and transferred onto PVDF membranes. The membranes were blocked with $5 \%$ non-fat milk at $25^{\circ} \mathrm{C}$ for $1 \mathrm{~h}$, and incubated with the aforementioned primary and secondary antibodies at $25^{\circ} \mathrm{C}$ for $1 \mathrm{~h}$, sequentially. Protein blots were developed using ECL western blotting substrate (Pierce; Thermo Fisher Scientific, Inc.) and the intensity of bands was quantified using ImageJ software (1.52v; National Institutes of Health).

TOP/FOP-flash reporter assay. A total of $50 \mathrm{ng}$ TOP/FOPFlash vectors (Promega Corporation) and 5 pmol miR-NC inhibitor or miR-934 inhibitor were co-transfected into 5,000 HCT116 cells using Lipofectamine 3000 (Invitrogen; Thermo Fisher Scientific, Inc.) at $37^{\circ} \mathrm{C}$ for $48 \mathrm{~h}$. The TOP/FOP ratio was measured to assess the activity of $\mathrm{Wnt} / \beta$-catenin signaling via GloMax 20 (Promega Corporation) $48 \mathrm{~h}$ post-transfection.

Cell proliferation and apoptosis assays. The proliferative ability of HCT116 cells was assessed using a Cell Counting Kit-8 (CCK-8; cat. no. CK04; Dojindo Molecular Technologies, Inc.). Cells were seeded into 96-well plates. CCK-8 solution $(10 \mu \mathrm{l})$ was added into each well and incubated for $2,0,24,48,72$ and $96 \mathrm{~h}$ after transfection. Cell proliferation was subsequently analyzed at a wavelength of $450 \mathrm{~nm}$. 
Cell apoptosis was assessed using the Annexin V-FITC/propidiumiodide (PI) apoptosis assay kit (cat.no. V13241; Invitrogen; Thermo Fisher Scientific, Inc.). Briefly, cells were harvested and suspended in Annexin $\mathrm{V}$ binding buffer. PI and Annexin V-FITC were added to the cells and incubated for $30 \mathrm{~min}$ at room temperature in the dark. Apoptotic cells were subsequently analyzed using a flow cytometer MACSQuant ${ }^{\circledR}$ X (Miltenyi Biotec $\mathrm{GmbH}$ ) and examined on FlowJo (version 10.7; FlowJo LLC). $\mathrm{PI}^{+} /$Annexin $\mathrm{V}^{+}$and $\mathrm{PI}^{-} /$Annexin $\mathrm{V}^{+}$were classified as apoptotic cells.

Bioinformatics analysis. The miR-934 expression data in 411 colon carcinoma samples and 380 normal mucosa samples were downloaded from the GSE115513 dataset using GEO2R (14) within the Gene Expression Omnibus database (www.ncbi.nlm.nih.gov/gds). GEO2R was also used to retrieve the top 250 differentially expressed miRNAs between normal and tumor tissues from GSE115513 with $\mathrm{P}<0.05$. The potential target genes of miR-934 were predicted using TargetScan software (version 7.2; www.targetscan.org/vert_72).

Dual-luciferase reporter assay. DDK2 3'UTR was amplified from cDNA of HCT116 and ligated into a pmirGLO plasmid (Promega Corporation). pmirGLO-DDK2 3'UTR-mutant (MUT) was constructed by introducing mutations into DDK2 3'UTR-wild-type (WT). Cells were transfected with DDK2 3'UTR-WT or DDK2 3'UTR-MUT in combination with miR-NC mimic or miR-934 mimic, using Lipofectamine 3000. After $48 \mathrm{~h}$, relative luciferase activity was detected using the Dual Luciferase Reporter System kit (cat. no. E1910; Promega Corporation). The firefly luciferase was normalized to Renilla luciferase activity.

Statistical analysis. Statistical analysis was performed using GraphPad Prism 6.0 software (GraphPad Software, Inc.). All experiments were performed in triplicate and data are presented as the mean \pm standard deviation. Pearson's correlation analysis was performed between miR-934 and DDK2 or c-Myc and cyclinD1 expression levels. Paired Student's t-test was used to compare differences between two groups of tissues, while unpaired Student's t-test was used to compare differences between two unpaired groups. One-way analysis of variance and Fisher's Least Significant Difference post hoc test were performed to compare differences between multiple groups. $\mathrm{P}<0.05$ was considered to indicate a statistically significant difference.

\section{Results}

miR-934 expression is upregulated in colorectal cancer. To investigate the tumor-associated miRNAs in colorectal cancer, previously published miRNA microarray data were analyzed. The top 250 differentially expressed miRNAs between colon carcinoma and normal mucosa were obtained; miR-934 was one of the most significantly upregulated miRNAs in colon carcinoma $(n=411)$ compared with normal mucosa $(n=380)$ (Fig. 1A). RT-qPCR analysis was performed to detect miR-934 expression in the tissue samples collected in the present study. Consistently, it was demonstrated that miR-934 expression was upregulated in colorectal tumor tissues compared with matched adjacent normal tissues from the 40 patients with colorectal cancer (Fig. 1B). In addition, miR-934 expression was higher in colorectal cancer cell lines (HCT116 and HT29) compared with that in the immortalized colon cells (FHC) (Fig. 1C).

DDK2 is a target gene of miR-934 in colorectal cancer cells. TargetScan software was used to predict the potential targets of miR-934. The results demonstrated that there was a putative binding site for miR-934 in the 3'UTR of DDK2 mRNA (Fig. 2A). RT-qPCR analysis demonstrated that DDK2 was downregulated in colorectal tumors (Fig. 2B). In addition, Pearson's correlation analysis revealed a negative correlation $(r=-0.456)$ between miR-934 and DDK2 expression levels in the collected tissue samples (Fig. 2C). Subsequently, HCT116 cells were transfected with miR-934 inhibitor to downregulate miR-934 expression (Fig. 2D). Downregulation of miR-934 increased DDK2 mRNA and protein levels in HCT116 cells (Fig. 2E and F). In addition, transfection with miR-934 mimic increased miR-934 expression in HCT116 cells compared with that in the miR-NC group (Fig. 2G). Overexpression of miR-934 decreased the relative luciferase activity of DDK2 3'UTR-WT, while the relative luciferase activity of DDK2 3'UTR-MUT was not affected by overexpression of miR-934 (Fig. 2H), suggesting that miR-934 directly interacted with DDK2 3'UTR in HCT116 cells. To validate this direct interaction, a RIP assay was performed. The results demonstrated that AGO2 enriched both DKK2 mRNA and miR-934 in HCT116 cells (Fig. 2I).

miR-934 activates the Wnt signaling pathway in colorectal cancer. DDK2 is a well-known negative regulator of Wnt signaling (18). Thus, $\beta$-catenin, the core effector of the Wnt signaling pathway (18), and $\mathrm{p}$ - $\beta$-catenin, the activated form of $\beta$-catenin, protein levels were assessed in HCT116 cells. Western blot analysis demonstrated that downregulation of miR-934 decreased p- $\beta$-catenin levels; however, it did not alter $\beta$-catenin expression in HCT116 cells (Fig. 3A). The results of the TOP/FOP assay demonstrated that the transcriptional activity of TOP/FOP was notably repressed in HCT116 cells following the downregulation of miR-934, suggesting that the Wnt signaling pathway was inactivated by the miR-934 inhibitor (Fig. 3B). Furthermore, downregulation of miR-934 decreased the mRNA expression levels of c-Myc and cyclin D1, which are well-known target genes of Wnt signaling (Fig. 3C) (18). Western blot analysis confirmed that downregulation of miR-934 also decreased c-Myc and cyclin D1 protein expression levels in HCT116 cells (Fig. 3D). RT-qPCR analysis was performed to detect c-Myc mRNA expression levels in the 40 tumor samples. The results demonstrated a notably positive correlation $(\mathrm{r}=0.823)$ between miR-934 and c-Myc mRNA expression in these tumors (Fig. 3E). A relatively weak positive correlation $(r=0.332)$ was also observed between miR-934 and cyclin D1 mRNA expression level in tumors (Fig. 3F).

DDK2 silencing rescues inactivation of Wnt signaling upon downregulation of miR-934 in colorectal cancer cells. DDK2 siRNA was transfected into HCT116 cells to downregulate DDK2 expression (Fig. 4A). As expected, DDK2 silencing 

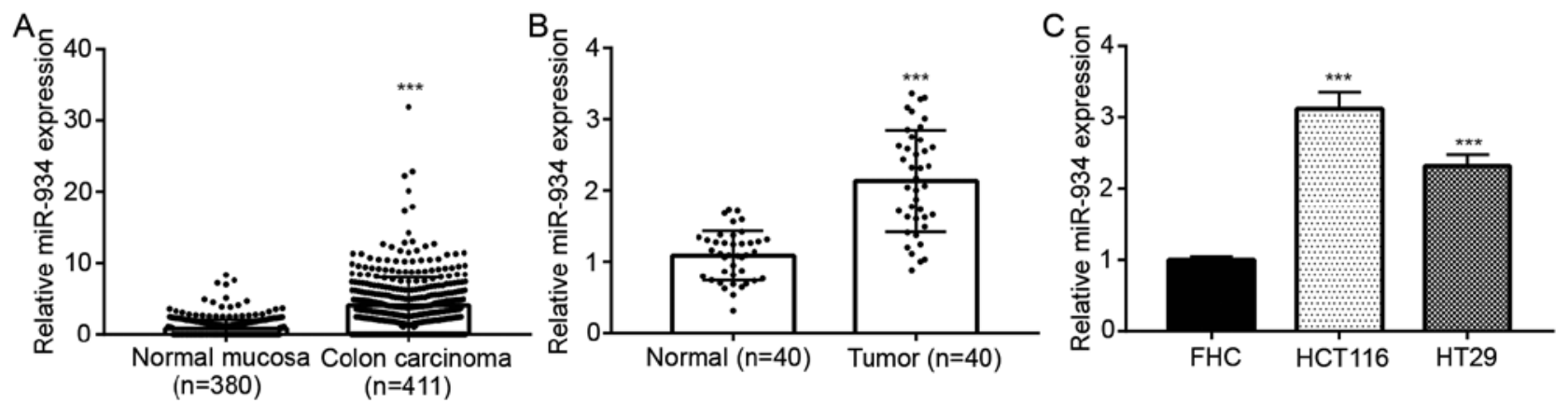

Figure 1. miR-934 is upregulated in colorectal cancer. (A) Analysis of GSE115513 dataset suggested that miR-934 was a significantly upregulated miRNA in colon carcinoma compared with normal mucosa. (B) In the collected tissues, miR-934 expression level was increased in colorectal tumors compared with that in matched normal tissues. (C) miR-934 expression level was increased in colorectal cancer cell lines HCT116 and HT29 compared with that in the immortalized human colon cell line FHC. ${ }^{* * *} \mathrm{P}<0.001$ vs. the respective control group. miR, microRNA.
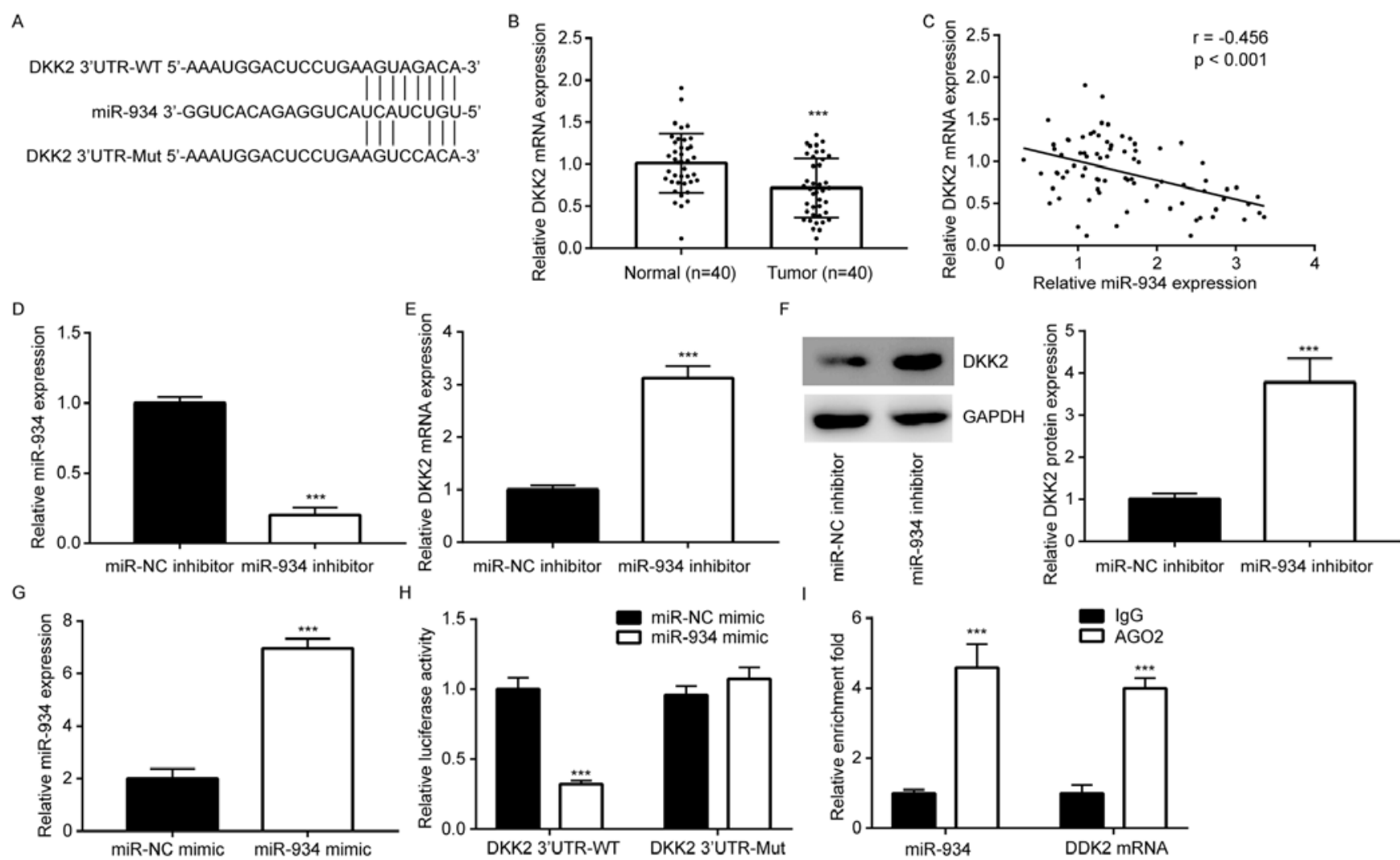

Figure 2. DKK2 is a target gene of miR-934. (A) Sequence alignment of miR-934 with DKK2 3'UTR-WT and DKK2 3'UTR-Mut. (B) DDK2 mRNA expression level was decreased in colorectal tumors compared with that in the matched normal tissues. (C) Pearson's correlation analysis suggested that there was a negative correlation between miR-934 and DKK2 mRNA expression in the collected tumor tissue samples. (D) Transfection of miR-934 inhibitor decreased miR-934 expression in HCT116 cells. (E) Transfection of miR-934 inhibitor increased DKK2 mRNA expression in HCT116 cells. (F) Transfection of miR-934 inhibitor increased DKK2 protein expression in HCT116 cells. (G) Transfection of miR-934 mimic increased miR-934 expression in HCT116 cells. (H) Overexpression of miR-934 repressed the relative luciferase activity of DDK2 3'UTR-WT in HCT116 cells. (I) RNA immunoprecipitation assay with AGO2 antibody was performed in HCT116 cells. Reverse transcription-quantitative PCR results showed that AGO2 enriched both DKK2 mRNA and miR-934 levels. ${ }^{* * *} \mathrm{P}<0.001$ vs. the respective control group. WT, wild-type; Mut, mutant; UTR, untranslated region; AGO2, protein argonaute-2; NC, negative control; DDK2, Dickkopf-related protein 2.

reversed the downregulation of $\mathrm{p}$ - $\beta$-catenin protein levels induced by miR-934 inhibitor in HCT116 cells (Fig. 4B). In addition, RT-qPCR analysis demonstrated that DDK2 silencing also reversed the decreased mRNA expression levels of c-Myc and cyclin D1, induced by miR-934 inhibitor in HCT116 cells (Fig. 4C). Taken together, these results suggest that miR-934 positively regulated Wnt signaling by targeting DKK2 in colorectal cancer cells.
miR-934/DKK2 axis controls cell proliferation and apoptosis in colorectal cancer cells. To determine the biological role of miR-934 in colorectal cancer, the CCK-8 assay was performed to assess the proliferative ability of HCT116 cells. The results demonstrated that miR-934 inhibition repressed the proliferation of HCT116 cells, which was reversed following DDK2 knockdown (Fig. 5A). Flow cytometric analysis demonstrated that miR-934 inhibition induced significant cell apoptosis 
A

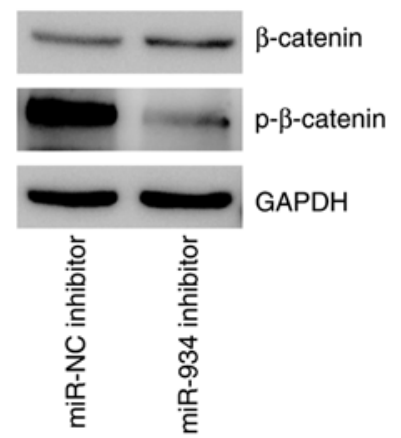

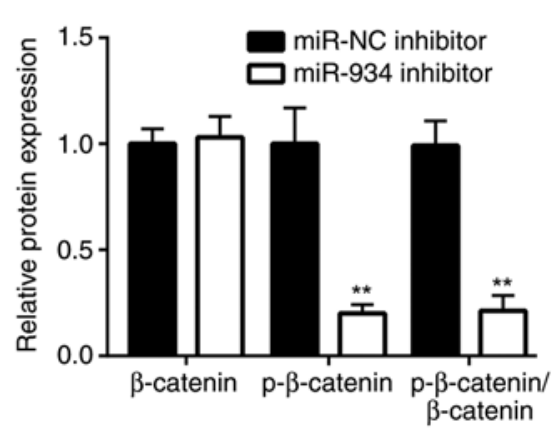

D

C

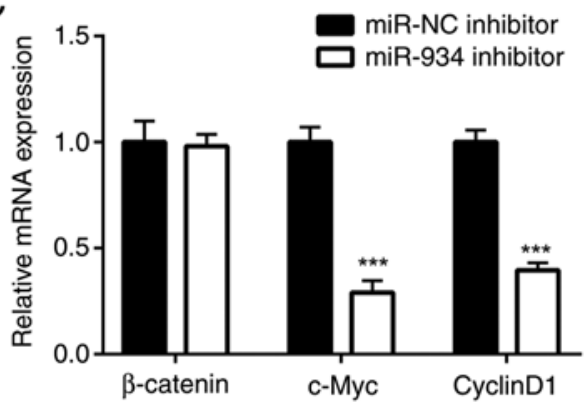

$\mathrm{E}$
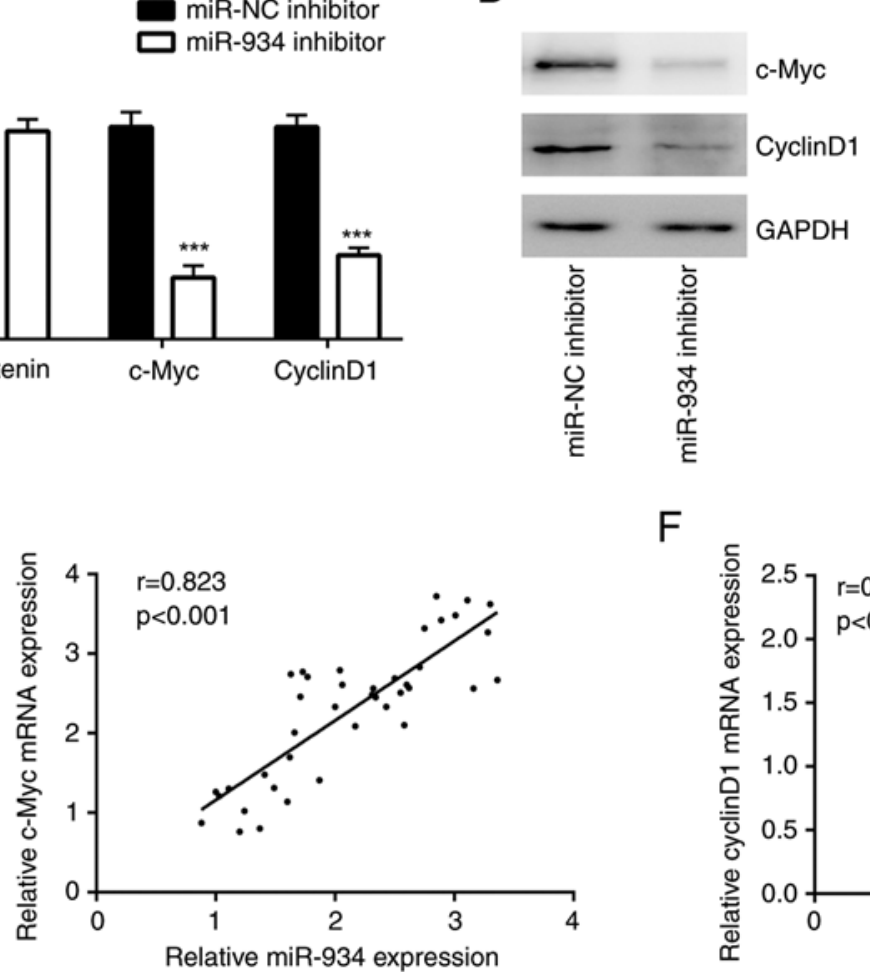

F
B
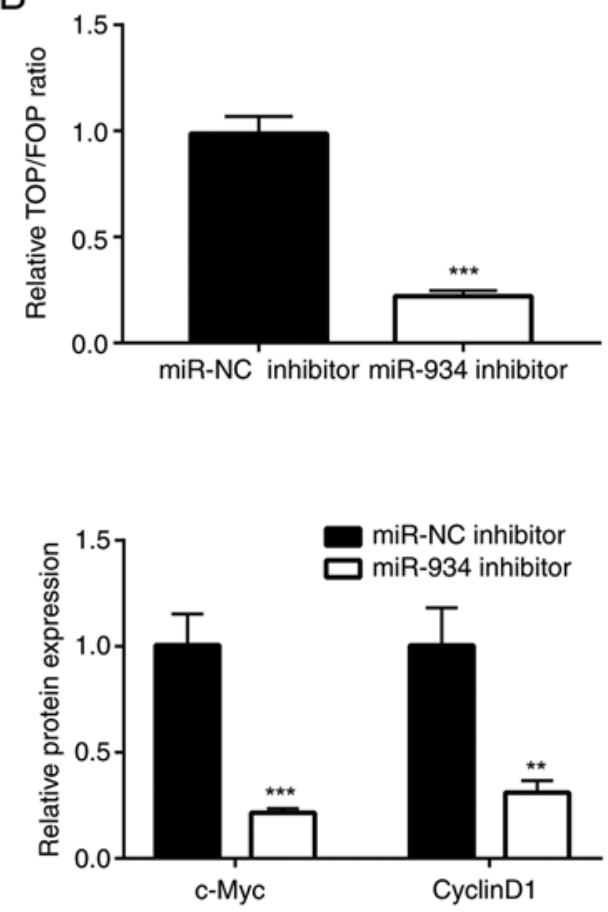

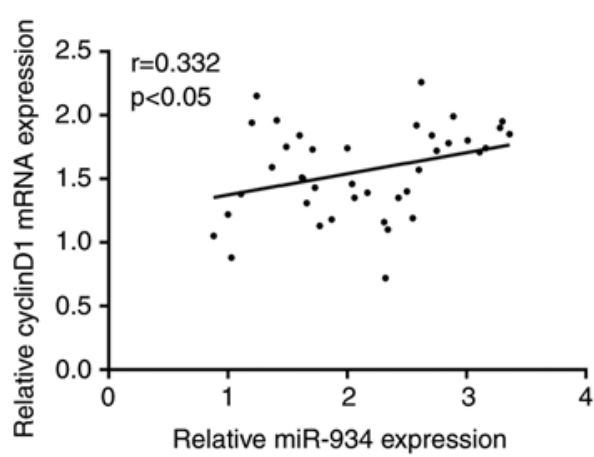

Figure 3. miR-934 downregulation inhibits Wnt signaling in HCT116 cells. (A) Western blotting showed that miR-934 downregulation decreased p- $\beta$-catenin protein levels in HCT116 cells. (B) TOP/FOP reporter assay was performed in HCT116 cells. The data showed that downregulation of miR-934 repressed the reporter activity in the cells. Downregulation of miR-934 decreased c-Myc and cyclinD1 (C) mRNA and (D) protein expression in HCT116 cells. Pearson's correlation analysis suggested that there was a negative correlation (E) between miR-934 and c-Myc mRNA expression and (F) between miR-934 and cyclinD1 mRNA expression in the collected tumor tissue samples. ${ }^{* *} \mathrm{P}<0.01$ and ${ }^{* * *} \mathrm{P}<0.001 \mathrm{vs}$. the miR-NC inhibitor group. miR, microRNA; NC, negative control; p, phosphorylated.

in HCT116 cells, which was also reversed following DDK2 knockdown (Fig. 5B). Collectively, these results suggest that the miR-934/DKK2 axis plays a role in regulating cell proliferation and apoptosis of colorectal cancer cells.

\section{Discussion}

Aberrant expression of miRNAs is responsible for the development of colorectal cancer (19-21). Previously, miR-934 was discovered as a cancer-associated miRNA via sequencing and experimental validation (14). RNA sequencing data suggested that miR-934 was one of eight miRNAs associated with alcohol-associated HNSCC, and the data from in vitro assays demonstrated that miR-934 facilitated HNSCC cell proliferation and resistance to apoptosis (16). In bladder cancer,
miR-934 promoted cell proliferation and cell cycle progression by targeting UBE2N and downregulating CDK6 (22). The results of the present study demonstrated that miR-934 was upregulated in colorectal cancer. Downregulation of miR-934 inhibited cell proliferation and induced the apoptosis of HCT116 cells. Collectively, these results suggest the oncogenic potential of miR-934 in colorectal cancer. However, the present study did not investigate the biological functions of miR-934 in colorectal cancer in vivo. Thus, future studies will aim to establish animal models to determine whether miR-934 promotes colorectal tumor growth and metastasis in vivo.

Wnt/ $\beta$-catenin signaling is crucial for cell proliferation, stemness, migration, invasion and resistance to apoptosis of colorectal cancer $(23,24)$. Upon activation, the transcription factor $\beta$-catenin is phosphorylated and translocated into the 


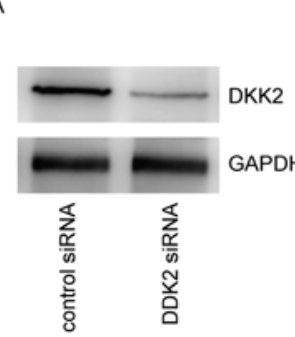

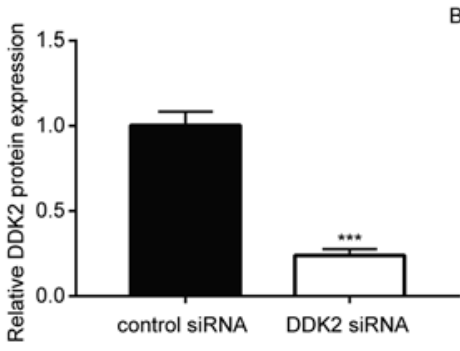

C

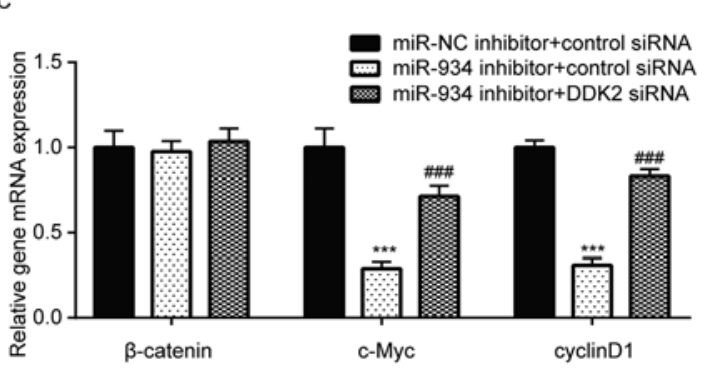

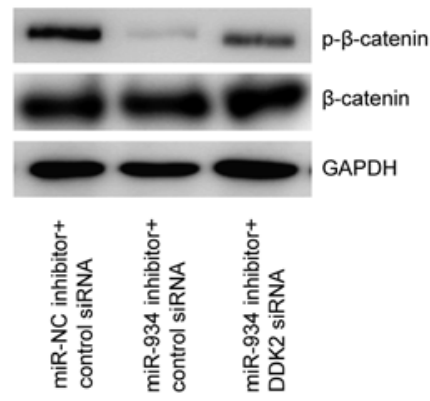

Figure 4. miR-934 regulates Wnt/ß-catenin signaling by targeting DKK2. (A) Transfection of DKK2 siRNA decreased the DKK2 protein expression level in HCT116 cells. ${ }^{* * *} \mathrm{P}<0.001$ vs. control siRNA. (B) DKK2 knockdown reversed downregulation of $\mathrm{p}-\beta$-catenin protein level induced by miR- 934 inhibitor in HCT116 cells. ${ }^{* * *} \mathrm{P}<0.001$ vs. miR-NC inhibitor + control siRNA and ${ }^{\# \#} \mathrm{P}<0.01$ vs. miR-934 inhibitor + control siRNA. (C) DKK2 knockdown reversed the downregulation of c-Myc and cyclinD1 mRNA expression levels induced by miR-934 inhibitor in HCT116 cells. ${ }^{* * *} \mathrm{P}<0.001$ vs. miR-NC inhibitor + control siRNA; ${ }^{\# \#} \mathrm{P}<0.001$ vs. miR-934 inhibitor + control siRNA. siRNA, small interfering RNA; miR, microRNA; NC, negative control; DDK2, Dickkopf-related protein 2.

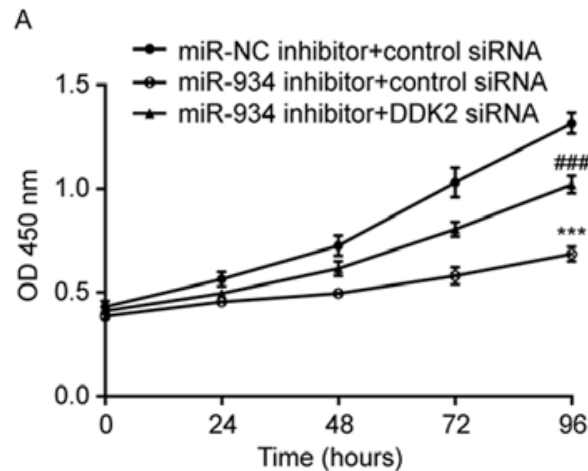

B

miR-NC inhibitor+control siRNA miR-934 inhibitor+control siRNA miR-934 inhibitor+DDK2 siRNA
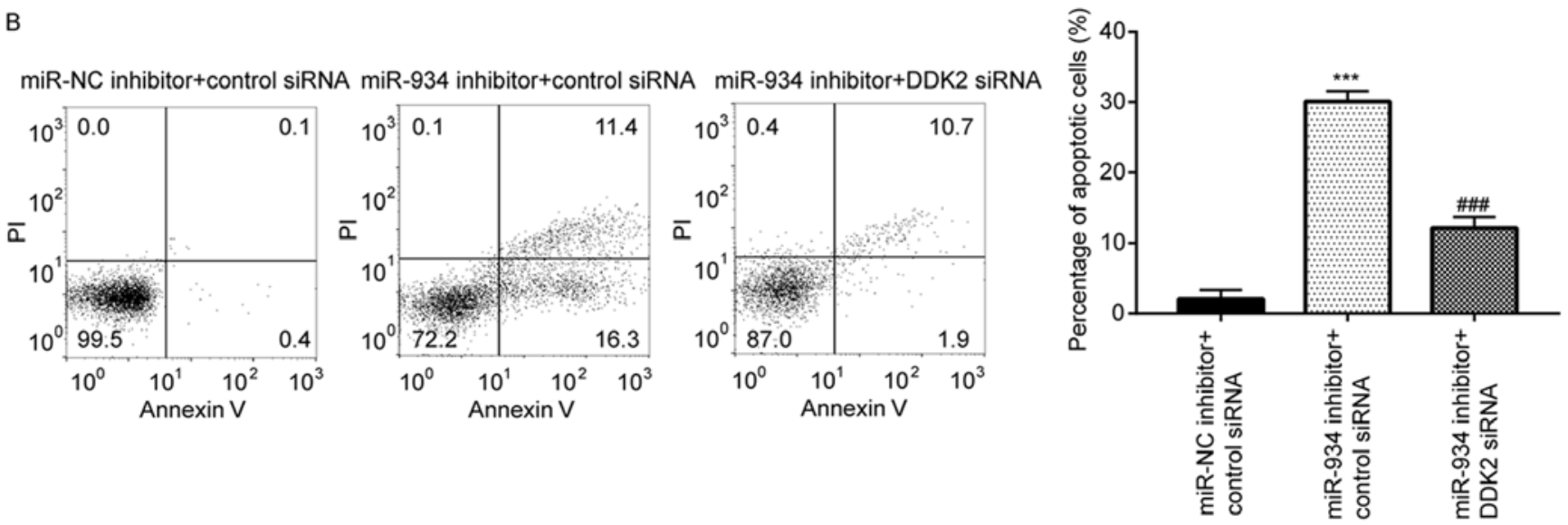

Figure 5. miR-934 regulated HCT116 cell proliferation and apoptosis by targeting DKK2. (A) Cell Counting Kit-8 assay results indicated that DKK2 knockdown reversed the inhibition of cell proliferation induced by miR-934 inhibitor in HCT116 cells. (B) Flow cytometry results indicated that DKK2 silencing reversed cell apoptosis induced by miR-934 inhibitor in HCT116 cells. ${ }^{* * *} \mathrm{P}<0.001$ vs. miR-NC inhibitor + control siRNA; \#\#" $\mathrm{P}<0.001$ vs. miR-934 inhibitor + control siRNA. siRNA, small interfering RNA; miR, microRNA; NC, negative control; DDK2, Dickkopf-related protein 2.

nucleus to activate transcription of its downstream target genes, such as c-Myc and cyclin D1 (25). DKK2 is an antagonist of canonical Wnt/ $\beta$-catenin signaling (26). Downregulation of DKK2 has been reported in several types of cancer, including 
colorectal cancer $(27,28)$. The present study identified DKK2 as a target gene of miR-934. In addition, the results demonstrated that miR-934 activated Wnt/ $\beta$-catenin signaling by targeting DKK2 in HCT116 cells. DKK2 has previously been reported as a target gene of miR-154 and miR-27a in in cardiac fibroblasts and bone marrow stromal cells $(29,30)$. Collectively, the results of the present study suggest that miR-934 may act as a novel regulator of DKK2. In addition, miR-934 expression was negatively associated with DKK2 mRNA expression in the collected patient tissue samples. DDK2 silencing reversed the biological function of miR-934 inhibition in HCT116 cells. Taken together, these results suggest that the miR-934/DKK2 axis is involved in proliferation and apoptosis of colorectal cancer cells.

In conclusion, the results of the present study demonstrated a role of miR-934 in mediating colorectal cancer cell proliferation by targeting DKK2, suggesting that miR-934 may be a potential biomarker for patients with colorectal cancer.

\section{Acknowledgements}

Not applicable.

\section{Funding}

No funding was received.

\section{Availability of data and materials}

The datasets used and/or analyzed during the current study are available from the corresponding author on reasonable request.

\section{Authors' contributions}

WL acquired the data. LM collected clinical samples. WL and JZ analyzed the data and wrote the manuscript. JZ supervised the study. All authors read and approved the final manuscript. WL and JZ confirm the authenticity of all the raw data.

\section{Ethics approval and consent to participate}

All procedures performed in the present study involving human participants were supervised and approved by the Ethics Committee of Shaanxi Provincial Cancer Hospital (approval no. 2015-23; Xi'an, China) and written informed consent was provided by all patients prior to the study commencement.

\section{Patient consent for publication}

Not applicable.

\section{Competing interests}

The authors declare that they have no competing interests.

\section{References}

1. Bray F, Ferlay J, Soerjomataram I, Siegel RL, Torre LA and Jemal A: Global cancer statistics 2018: GLOBOCAN estimates of incidence and mortality worldwide for 36 cancers in 185 countries. CA Cancer J Clin 68: 394-424, 2018.
2. Joachim C, Macni J, Drame M, Pomier A, Escarmant P, Veronique-Baudin $\mathrm{J}$ and Vinh-Hung V: Overall survival of colorectal cancer by stage at diagnosis: Data from the Martinique Cancer Registry. Medicine (Baltimore) 98: e16941, 2019.

3. Diao F and Cai S: Aspirin-based chemoprevention of colorectal cancer: The role for gut microbiota. Cancer Commun (Lond) 40 : 633-635, 2020.

4. Wang Y, Lin R, Ling H, Ke Y, Zeng Y, Xiong Y, Zhou Q, Zhou F and Zhou Y: Dual inhibition of CDK4 and FYN leads to selective cell death in KRAS-mutant colorectal cancer. Signal Transduct Target Ther 4: 52, 2019.

5. Liu S, Lin H, Wang D, Li Q, Luo H, Li G, Chen X, Li Y, Chen P, Zhai $\mathrm{B}$, et al. PCDH17 increases the sensitivity of colorectal cancer to 5-fluorouracil treatment by inducing apoptosis and autophagic cell death. Signal Transduct Target Ther 4: 53, 2019.

6. Bartel DP: MicroRNAs: Genomics, biogenesis, mechanism, and function. Cell 116: 281-297, 2004.

7. Bartel DP: MicroRNAs: Target recognition and regulatory functions. Cell 136: 215-233, 2009.

8. Alvarez-Garcia I and Miska EA: MicroRNA functions in animal development and human disease. Development 132: 4653-4662, 2005.

9. Yu D, Han GH, Zhao X, Liu X, Xue K, Wang D and Xu CB: MicroRNA-129-5p suppresses nasopharyngeal carcinoma lymphangiogenesis and lymph node metastasis by targeting ZIC2. Cell Oncol (Dordr) 43: 249-261, 2020.

10. Jacob H, Stanisavljevic L, Storli KE, Hestetun KE, Dahl O and Myklebust MP: Identification of a sixteen-microRNA signature as prognostic biomarker for stage II and III colon cancer. Oncotarget 8: 87837-87847, 2017.

11. Li Y, Zhuo ZJ, Zhou H, Liu J, Xiao Z, Xiao Y, He J and Liu Z: $\mathrm{miR}-34 \mathrm{~b} / \mathrm{c}$ rs4938723 T>C decreases neuroblastoma risk: A replication study in the hunan children. Dis Markers 2019: 6514608, 2019.

12. Li S, Wu X, Xu Y, Wu S, Li Z, Chen R, Huang N, Zhu Z and $\mathrm{Xu}$ X: miR-145 suppresses colorectal cancer cell migration and invasion by targeting an ETS-related gene. Oncol Rep 36: 1917-1926, 2016.

13. Huang W, Yan Y, Liu Y, Lin M, Ma J, Zhang W, Dai J, Li J, Guo Q, Chen H, et al: Exosomes with low miR-34c-3p expression promote invasion and migration of non-small cell lung cancer by upregulating integrin $\alpha 2 \beta 1$. Signal Transduct Target Ther 5: 39, 2020.

14. Slattery ML, Herrick JS, Pellatt DF, Stevens JR, Mullany LE, Wolff E, Hoffman MD, Samowitz WS and Wolff RK: MicroRNA profiles in colorectal carcinomas, adenomas and normal colonic mucosa: Variations in miRNA expression and disease progression. Carcinogenesis 37: 245-261, 2016.

15. Hu Y, Zhang Q, Cui J, Liao ZJ, Jiao M, Zhang YB, Guo YH and Gao YM: Oncogene miR-934 promotes ovarian cancer cell proliferation and inhibits cell apoptosis through targeting BRMS1L. Eur Rev Med Pharmacol Sci 23: 5595-5602, 2019.

16. Saad MA, Kuo SZ, Rahimy E, Zou AE, Korrapati A, Rahimy M, Kim E, Zheng H, Yu MA, Wang-Rodriguez J and Ongkeko WM: Alcohol-dysregulated miR-30a and miR-934 in head and neck squamous cell carcinoma. Mol Cancer 14: 181, 2015.

17. Livak KJ and Schmittgen TD: Analysis of relative gene expression data using real-time quantitative PCR and the 2(-Delta Delta C(T)) method. Methods 25: 402-408, 2001.

18. Hirata H, Hinoda Y, Nakajima K, Kawamoto K, Kikuno N, Kawakami K, Yamamura S, Ueno K, Majid S, Saini S, et al: Wnt antagonist gene DKK2 is epigenetically silenced and inhibits renal cancer progression through apoptotic and cell cycle pathways. Clin Cancer Res 15: 5678-5687, 2009.

19. Zheng YB, Xiao K, Xiao GC, Tong SL, Ding Y, Wang QS, Li SB and Hao ZN: MicroRNA-103 promotes tumor growth and metastasis in colorectal cancer by directly targeting LATS 2 . Oncol Lett 12: 2194-2200, 2016.

20. Zhao D, Ma Y, Li X and Lu X: microRNA-211 promotes invasion and migration of colorectal cancer cells by targeting FABP4 via PPAR $\gamma$. J Cell Physiol: Feb 26, 2019. doi: 10.1002/jcp.28190. (Epub ahead of print).

21. Zhang Z, Zhong X, Xiao Y and Chen C: MicroRNA-296 inhibits colorectal cancer cell growth and enhances apoptosis by targeting ARRB1-mediated AKT activation. Oncol Rep 41: 619-629, 2019.

22. Yan H, Ren S, Lin Q, Yu Y, Chen C, Hua X, Jin H, Lu Y, Zhang H, Xie Q, et al: Inhibition of UBE2N-dependent CDK6 protein degradation by miR-934 promotes human bladder cancer cell growth. FASEB J 33: 12112-12123, 2019. 
23. Chen G, Gao C, Gao X, Zhang DH, Kuan SF, Burns TF and Hu J: Wnt/ $\beta$-catenin pathway activation mediates adaptive resistance to BRAF inhibition in colorectal cancer. Mol Cancer Ther 17: 806-813, 2018

24. Bahrami A, Amerizadeh F, ShahidSales S, Khazaei M, Ghayour-Mobarhan M, Sadeghnia HR, Maftouh M, Hassanian SM and Avan A: Therapeutic potential of targeting Wnt/ $\beta$-catenin pathway in treatment of colorectal cancer: Rational and progress. J Cell Biochem 118: 1979-1983, 2017.

25. Kleszcz R, Szymanska A, Krajka-Kuzniak V, Baer-Dubowska W and Paluszczak J: Inhibition of $\mathrm{CBP} / \beta$-catenin and porcupine attenuates Wnt signaling and induces apoptosis in head and neck carcinoma cells. Cell Oncol (Dordr) 42: 505-520, 2019.

26. Zhu J, Zhang S, Gu L and Di W: Epigenetic silencing of DKK2 and Wnt signal pathway components in human ovarian carcinoma. Carcinogenesis 33: 2334-2343, 2012.

27. Kawakita A, Yanamoto S, Yamada S, Naruse T, Takahashi H, Kawasaki G and Umeda M: MicroRNA-21 promotes oral cancer invasion via the Wnt/ $\beta$-catenin pathway by targeting DKK2. Pathol Oncol Res 20: 253-261, 2014.
28. Deng F, Zhou R, Lin C, Yang S, Wang H, Li W, Zheng K, Lin W, Li X, Yao X, et al: Tumor-secreted dickkopf 2 accelerates aerobic glycolysis and promotes angiogenesis in colorectal cancer. Theranostics 9: 1001-1014, 2019.

29. Sun LY, Bie ZD, Zhang CH, Li H, Li LD and Yang J: MiR-154 directly suppresses DKK2 to activate Wnt signaling pathway and enhance activation of cardiac fibroblasts. Cell Biol Int 40: 1271-1279, 2016

30. Wu X, Gu Q, Chen X, Mi W, Wu T and Huang H: MiR-27a targets DKK2 and SFRP1 to promote reosseointegration in the regenerative treatment of peri-implantitis. J Bone Miner Res 34: 123-134, 2019.

\footnotetext{
c) (i) $\ominus$ This work is licensed under a Creative Commons Attribution-NonCommercial-NoDerivatives 4.0 International (CC BY-NC-ND 4.0) License.
} 